\title{
Bromodomain inhibition reveals FGF15/19 as a target of epigenetic regulation and metabolic control
}

Chisayo Kozuka ${ }^{1,2,3}$, Vissarion Efthymiou ${ }^{1,2}$, Vicencia M. Sales ${ }^{1,2}$, Liyuan Zhou ${ }^{1,4}$, Soravis Osataphan ${ }^{1,2}$, Yixing Yuchi ${ }^{1,2}$, Jeremy Chimene-Weiss ${ }^{1}$, Christopher Mulla ${ }^{1,2}$, Elvira Isganaitis $^{1,2}$, Jessica Desmond ${ }^{1}$, Suzuka Sanechika ${ }^{1}$, Joji Kusuyama ${ }^{1,2}$, Laurie Goodyear $^{1,2}$, Xu Shi ${ }^{2,4}$, Robert E. Gerszten ${ }^{2,4}$, Cristina Aguayo-Mazzucato ${ }^{1,2}$, Priscila Carapeto $^{1,2}$, Silvania DaSilva Teixeira ${ }^{6}$, Darleen Sandoval ${ }^{6}$, Direna Alonso-Curbelo ${ }^{7}$, Lei $\mathrm{Wu}^{2,5}$, Jun $\mathrm{Qi}^{2,5}$, and Mary-Elizabeth Patti ${ }^{1,2, *}$

\section{Supplemental material}

\section{Hyperinsulinemic-euglycemic clamp}

Hyperinsulinemic-euglycemic clamp was conducted in conscious mice as previously described (1). Briefly, following basal assessment, a primed, continuous infusion (150 $\mathrm{mU} / \mathrm{kg}$ body weight bolus, then $1.25 \mathrm{mU} / \mathrm{kg} / \mathrm{min}$ ) of human insulin was initiated. At the end of the clamp, a bolus of ${ }^{14} \mathrm{C}$ deoxyglucose was administered prior to sacrifice and tissue dissection.

\section{Metabolomics analysis}

Plasma and liver samples obtained at sacrifice (day16) were used for analysis of plasma and liver metabolites, using liquid chromatography-mass spectrometry (LC/MS) to determine metabolites, lipid class, and specific lipid content (2). Missing data were imputed with half of the minimum intensity of the metabolite, and the imputed data were quantile normalized and $\log _{2}$-transformed. 


\section{Pancreatic islet isolation and glucose-stimulated insulin secretion}

Pancreatic islets were isolated as previously described (3). For insulin secretion assay, islets were isolated from 5 male C57BL/6 mice and cultured for 72 hours in RPMI-1640 medium (ThermoFisher Scientific) with or without JQ-1 (80 or $250 \mathrm{nM}$ ). Insulin secretion was measured by incubation in $2.9 \mathrm{mM}$ or $20.2 \mathrm{mM}$ glucose in Krebs-Ringer bicarbonate buffer.

\section{Primary hepatocyte isolation and measurement of glucose production}

Primary hepatocytes were isolated from C57BL/6 mice after liver perfusion with collagenase and seeded $1 \times 10^{5}$ cells $/ \mathrm{ml}$ into collagen coated plates containing DMEM (ThermoFisher Scientific). The media were changed after 4 hours and glucose production was measured the following day as previously described (4). Briefly, after $6 \mathrm{~h}$ serum starvation with or without JQ-1 and FGF19, cells were cultured in glucose and phenol red-free DMEM with or without insulin (10, $100 \mathrm{nM}), \mathrm{JQ}-1$ (250 nM) and FGF19 (100 $\mathrm{ng} / \mathrm{ml})$ for $5 \mathrm{~h}$. Medium glucose concentrations were measured with the Glucose (HK) Assay Kit (Sigma). 


\section{Supplemental Reference}

1. Kim JK: Hyperinsulinemic-euglycemic clamp to assess insulin sensitivity in vivo. Methods Mol Biol 2009;560:221-238

2. Roberts LD, Souza AL, Gerszten RE, Clish CB: Targeted metabolomics. Curr Protoc Mol Biol 2012; Chapter 30:Unit 3032 31-24

3. Aguayo-Mazzucato C, Andle J, Lee TB, Jr., Midha A, Talemal L, Chipashvili V, Hollister-Lock J, van Deursen J, Weir G, Bonner-Weir S: Acceleration of beta Cell Aging Determines Diabetes and Senolysis Improves Disease Outcomes. Cell Metab 2019;30:129-142 e124

4. Matsumoto M, Sakai M: Glucose Production Assay in Primary Mouse Hepatocytes. Bio-protocol 2012;2:e284 
Supplemental Table 1. Transcriptome pathway analysis of liver in JQ1-treated mice Supplemental Table 2. Primer sequences

\section{Mouse}

\begin{tabular}{|c|c|c|}
\hline Gene & Forward Sequence & Reverse Sequence \\
\hline Rp/13a & CTGCTCTCAAGGTTGTTCGGCT & CCTTCCGTTTCTCCTCCAGAGT \\
\hline Slc10a2 (ASBT) & GTCTGTCCCCCAAATGCAACT & CACCCCATAGAAAACATCACCA \\
\hline Gpbar1 (TGR5) & TGCTTCTTCCTAAGCCTACTACT & CTGATGGTTCCGGCTCCATAG \\
\hline Nr1h4 (FXR) & CTGGCATCTATGAACTCAGGC & CCATTCGCGGCTTCTTTGG \\
\hline Rxra & ATGGACACCAAACATTTCCTGC & CCAGTGGAGAGCCGATTCC \\
\hline Fgf15 & ACGTCCTTGATGGCAATCG & GAGGACCAAAACGAACGAAATT \\
\hline NrOb2 (SHP) & AGGATGCTGTGACCTTCGAG & CAGCTCAAGGCTCCAGAAAGA \\
\hline Slc51a (OSTa) & ATGCATCTGGGTGAACAGAA & GAGTAGGGAGGTGAGCAAGC \\
\hline Slc51b (OST $\beta)$ & GACCACAGTGCAGAGAAAGC & CTTGTCATCACCACCAGGAC \\
\hline Pck1 (PEPCK) & CTAACTTGGCCATGATGAACC & CTTCACTGAGGTGCCAGGAG \\
\hline G6pc & CTTTGACTCTCTGAAGCCCC & GGGCTAGGCAGTATGGGATA \\
\hline$A c c$ & CCTGAAGACCTTAAAGCCAATGC & CCAGCCCACACTGCTTGTA \\
\hline Fasn & GCCCGGGTAGCTCTGGGTGTA & TGCTCCCAGCTGCAGGC \\
\hline Ppara & CTTCCCAAAGCTCCTTCAAAAA & CTGCGCATGCTCCGTG \\
\hline Cpt1a & GCACTGCAGCTCGCACATTACAA & CTCAGACAGTACCTCCTTCAGGAA \\
\hline Srebp2 & GAACTTTTCCTTAACGTGGGCCT & GAGCATGTCTTCGATGTCGTTCA \\
\hline Hmgcs1 & AACTGGTGCAGAAATCTCTAGC & GGTTGAATAGCTCAGAACTAGCC \\
\hline $\mathrm{Hmgcr}$ & AGCTTGCCCGAATTGTATGTG & TCTGTTGTGAACCATGTGACTTC \\
\hline Nr1h3 (LXR) & AGGAGTGTCGACTTCGCAAA & CTCTTCTTGCCGCTTCAGTTT \\
\hline Cур7a1 & GAACCTCCTTTGGACAACGGG & GGAGTTTGTGATGAAGTGGACAT \\
\hline Cyp8b1 & TGTCTACTCCCTACTGGGGC & GAGAGCCACCTTATCTCCGC \\
\hline Fgfr1c & TGTTTGACCGGATCTACACACA & CTCCCACAAGAGCACTCCAA \\
\hline Fgfr2 & TCGCATTGGAGGCTATAAGG & CGGGACCACACTTTCCATAA \\
\hline Fgfr3 & GCCCGGAGCGAATGGATAAG & GGATGCTGCCAAACTTGTTCT \\
\hline Fgfr4 & CGCCAGCCTGTCACTATACAAA & CCAGAGGACCTCGACTCCAA \\
\hline$K l b$ & TGTTCTGCTGCGAGCTGTTAC & TACCGGACTCACGTACTGTTT \\
\hline Krt20 & CCTGCGAATTGACAATGCTA & CCTTGGAGATCAGCTTCCAC \\
\hline Hnf1a & GACCTGACCGAGTTGCCTAAT & CCGGCTCTTTCAGAATGGGT \\
\hline ChgA & АTCCTCTCTATCCTGCGACAC & GGGCTCTGGTTCTCAAACACT \\
\hline Muc2 & AACGATGCCTACACCAAGGTC & ACTGAACTGTATGCCTTCCTCA \\
\hline Lgr5 & CCTACTCGAAGACTTACCCAGT & GCATTGGGGTGAATGATAGCA \\
\hline
\end{tabular}




\begin{tabular}{|l|l|l|} 
Reg4 & CTGGAATCCCAGGACAAAGAGTG & CTGGAGGCCTCCTCAATGTTTGC \\
\hline Ctnnb1 (ß-catenin) & GCGGCCGCGAGGTACCTGAA & GCAGCTTTTCTGTCCGGCTCCA \\
\hline Gcg & AGGGACCTTTACCAGTGATGT & GCGAATGGCGACTTCTTCTGGGAA \\
\hline Pyy & ACGGTCGCAATGCTGCTAAT & GACATCTCTTTTTCCATACCGCT \\
\hline Crp4 & AAGAGACTAAAACTGAGGAGCAGC & CGGCGGGGGCAGCAGTA \\
\hline
\end{tabular}

Human

\begin{tabular}{|l|l|l|}
\hline \multicolumn{1}{|c|}{ Human Gene } & \multicolumn{1}{c|}{ Forward Sequence } & \multicolumn{1}{c|}{ Reverse Sequence } \\
\hline BRD4 & CATGGACATGAGCACAATCA & TCATGGTCAGGAGGGTTGTA \\
\hline MYC & GGCTCCTGGCAAAAGGTA & CTGCGTAGTTGTGCTGATGT \\
\hline FGF19 & CGGAGGAAGACTGTGCTTTCG & CTCGGATCGGTACACATTGTAG \\
\hline NROB2 (SHP) & ATCCTCTTCAACCCCGATGTG & ACTTCACACAGCACCCAGTG \\
\hline
\end{tabular}

ChIP-PCR

\begin{tabular}{|l|l|l|}
\hline ChIP-PCR primers & \multicolumn{1}{|c|}{ Forward Sequence } & \multicolumn{1}{c|}{ Reverse Sequence } \\
\hline FGF19-1 & GCTGGGACAAAGCATCGGA & TCCCAAAGGACAAGCCAAGTAA \\
\hline FGF19-2 & GTCCCGCTGTTCACAAAGGA & CCACCCCAGAAAGGATGCAG \\
\hline FGF19-3 & GTGCGGTCGGTCCACAA & TTTTGGCACCTGGACGTTAG \\
\hline SHP & TGGCTGGTGCTCATGGTTAG & TAAATAGCACCCACAGCGCA \\
\hline
\end{tabular}


Supplemental Figure 1. JQ-1 decreases insulin content in islets isolated from mice treated in vivo, and reduces glucose stimulated insulin secretion after ex vivo incubation with JQ1.
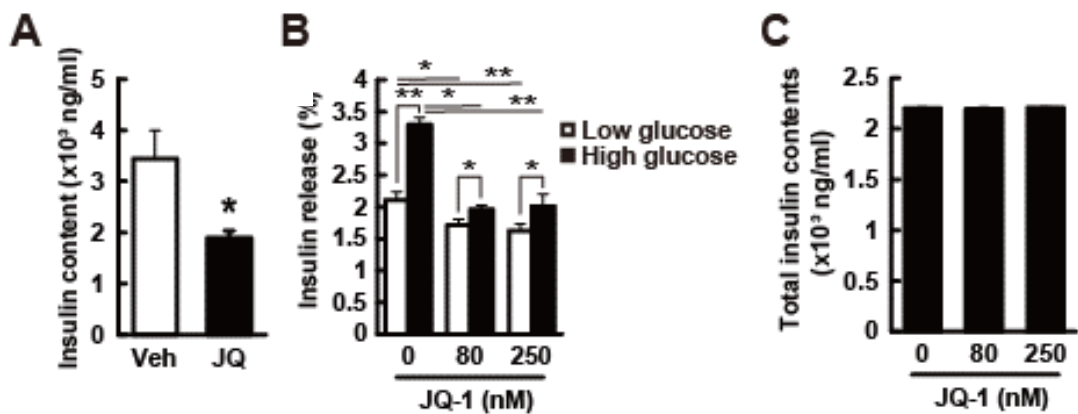

(A) Islet insulin content in pancreatic islets of JQ-1-treated mice. (B,C) Islets from 5 male C57BL/6 mice were divided into 3 groups: vehicle (Veh), JQ-1 at $80 \mathrm{nM}$ and $250 \mathrm{nM}$. (B) Glucose-stimulated insulin secretion (GSIS) under low (2.9 mM) and high (20.2 mM) glucose Krebs-Ringer solutions (after 1h incubation). (C) Total insulin content. Islets of each group were isolated in triplicate. 20 islets per sample; 3 samples per animal. Data are expressed as means \pm SEM. 
Supplemental Figure 2. JQ-1 has no effect on systemic or tissue insulin sensitivity.
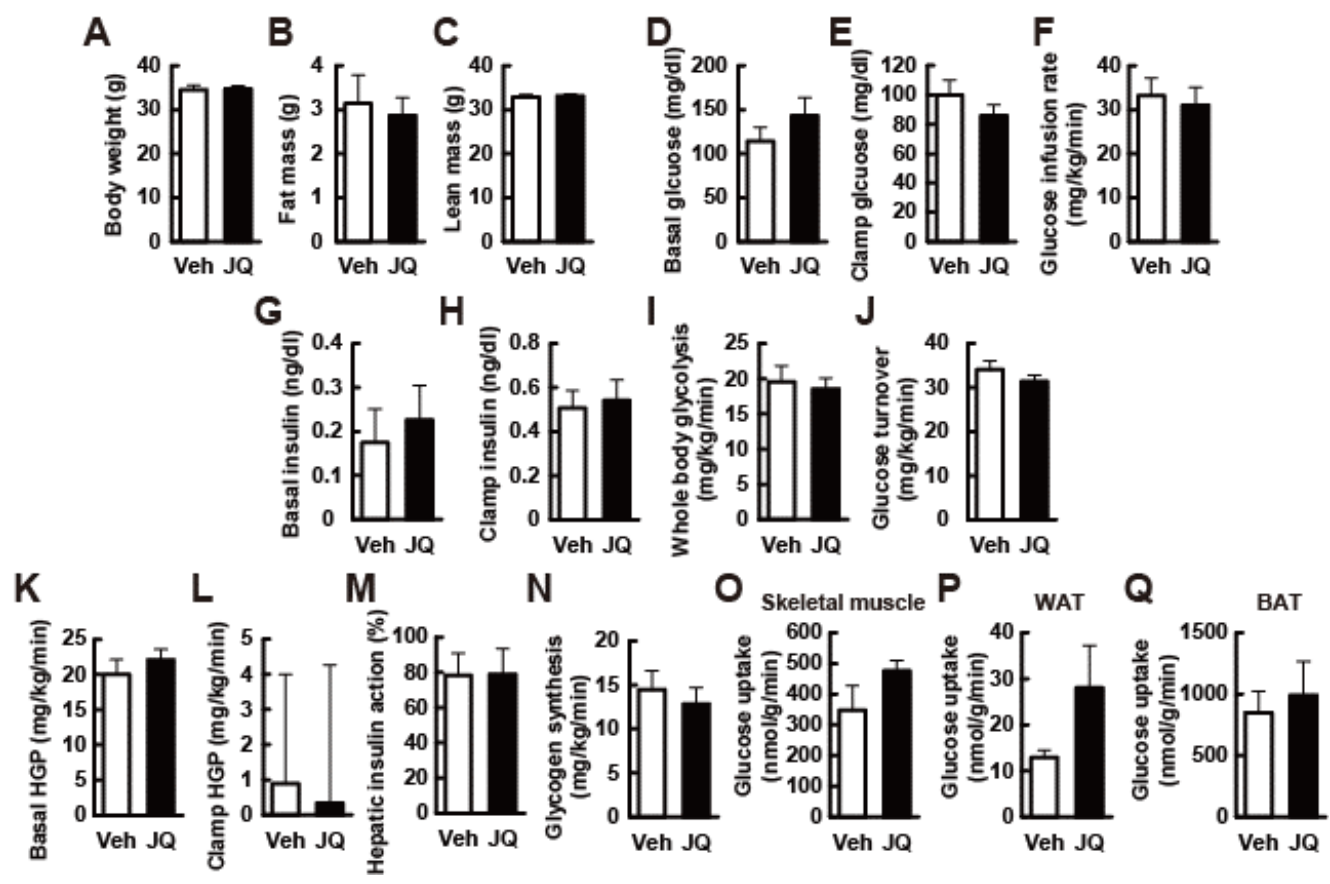

Hyperinsulinemic-euglycemic clamp experiments were performed to assess insulin sensitivity (1.25 mU/kg/min insulin dose) $(n=5-6)$. (A) Body weight, (B) fat mass, and (C) lean mass. (D) Basal glucose, (E) clamp glucose, (F) glucose infusion rate, (G) basal insulin, $(\mathbf{H})$ clamp insulin, (I) whole body glycolysis, and $(\mathbf{J})$ glucose turnover, $(\mathbf{K})$ basal hepatic glucose production (HGP), (L) clamp HGP, (M) hepatic insulin action, (N) glycogen synthesis. (O-Q) Glucose uptake in skeletal muscle (0), white adipose tissue $(\mathrm{WAT})(\mathbf{P})$, and brown adipose tissue $(\mathrm{BAT})(\mathbf{Q})$. Data are expressed as means \pm SEM. 
Supplemental Figure 3. JQ-1 has minimal effect on adipose tissue and skeletal muscle.
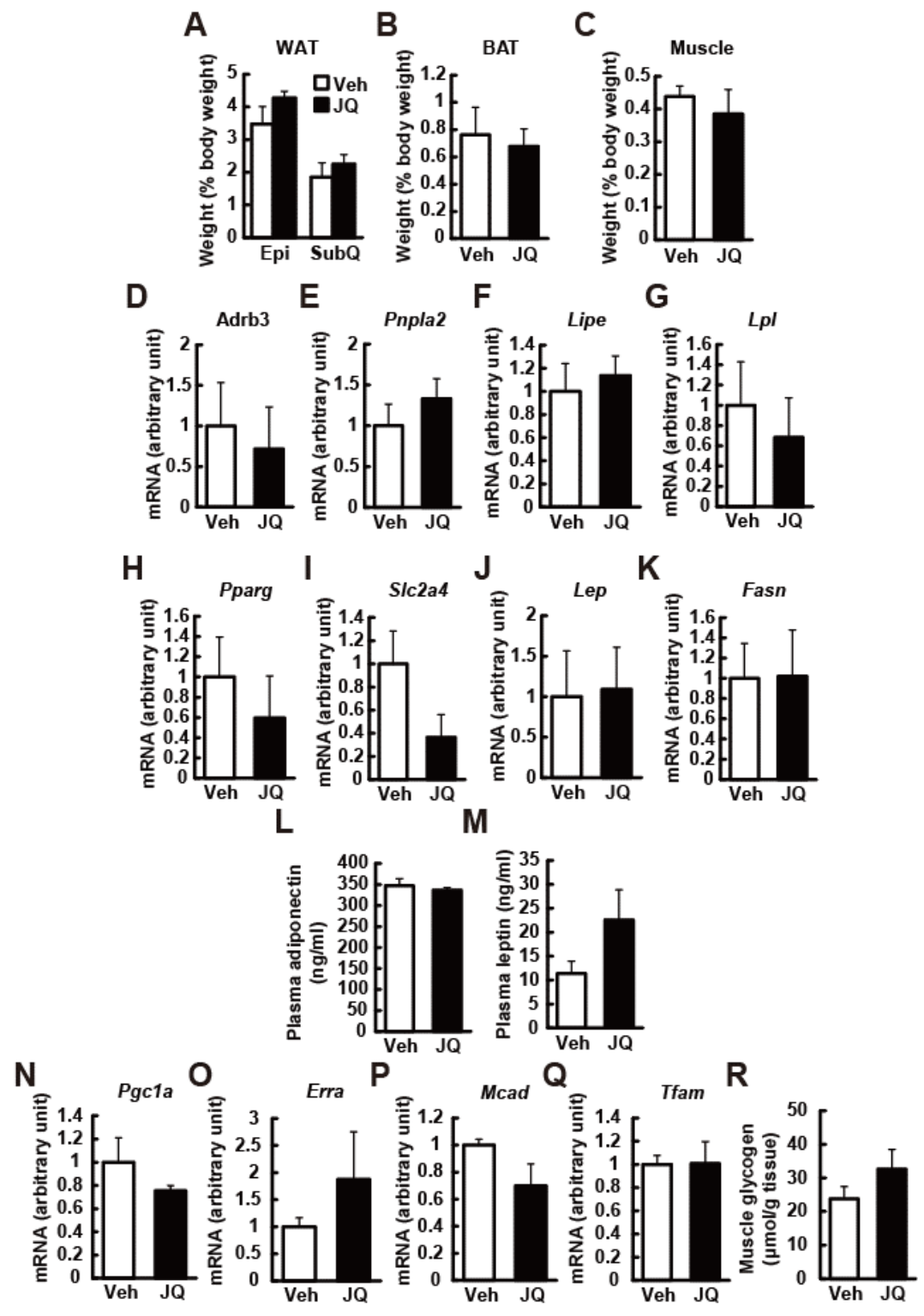

(A-C) Tissue weight of white adipose tissue (WAT) (A), brown adipose tissue (BAT) (B), and skeletal muscle (C) ( $n=5-6)$. Veh: Vehicle-treated mice, Epi: epididymal fat, SubQ: subcutaneous fat. (D-K) Epididymal adipose tissue expression of $A d r b 3$ ( $\beta 3-A d r R)$ (D), Pnpla2 (ATGL) (E), Lipe (HPL) (F), Lpl (G), Pparg (H), Slc2a4 (GLUT4) (I), Lep (J), and Fasn (K) ( $n=5-6)$. (L, M) Plasma adiponectin (L) and leptin (M) levels ( $n=5-6)$. (N-Q)

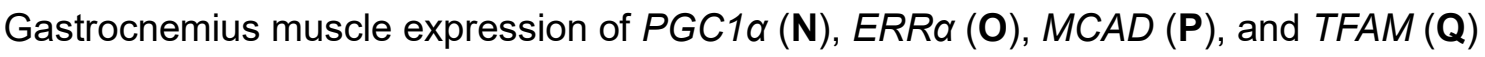
$(n=5-6)$. Gene expression levels were normalized to Rp/13. (R) Glycogen contents in gastrocnemius muscle $(n=5-6)$. Data are expressed as mean \pm SEM. 
Supplemental Figure 4. JQ-1 does not alter insulin signaling in liver extracts or either primary or cultured hepatocytes.

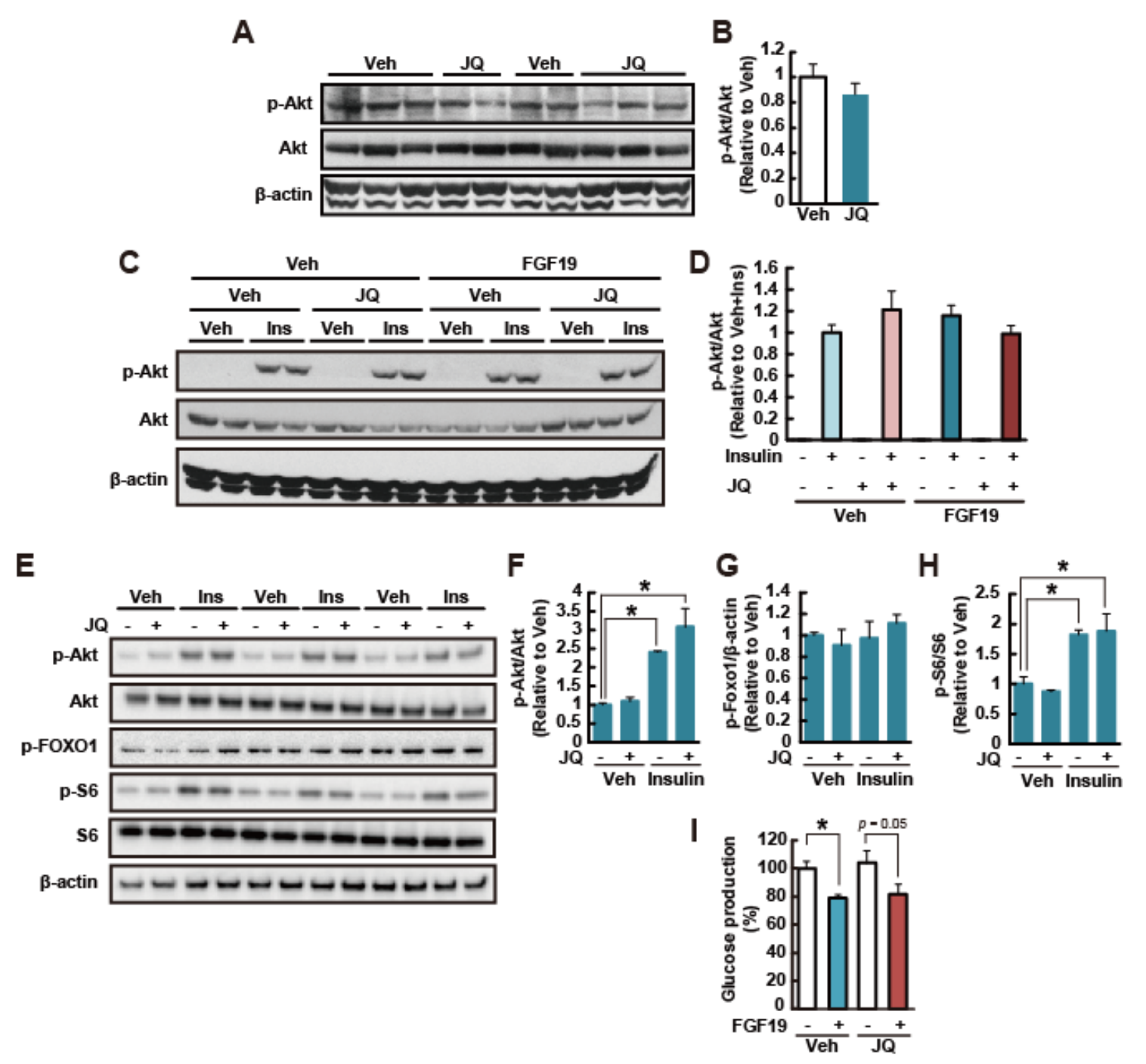

(A, B) Western blot analysis of total and phosphorylated Akt in liver lysates. $\beta$-actin is shown as a loading control $(n=5)$. (C-H) Western blot analysis of total and phosphorylated Akt (C-F), FOXO1 (E, G), and S6 (E, H) in primary hepatocytes (C, D) and AML12 cells $(\mathbf{E}-\mathbf{H})(n=3)$. $\beta$-actin is shown as a loading control. ${ }^{*} P<0.05$, vs. vehicle (Veh) or JQ-1-treated cells without insulin or FGF19. (I) Glucose production with or without FGF19 in primary hepatocytes $(n=3)$. ${ }^{*} P<0.05$, vs. vehicle (Veh) or JQ-1treated cells without FGF19. 


\section{Supplemental Figure 5. JQ-1 modulates expression of genes regulating glucose}

and lipid metabolism in the liver.

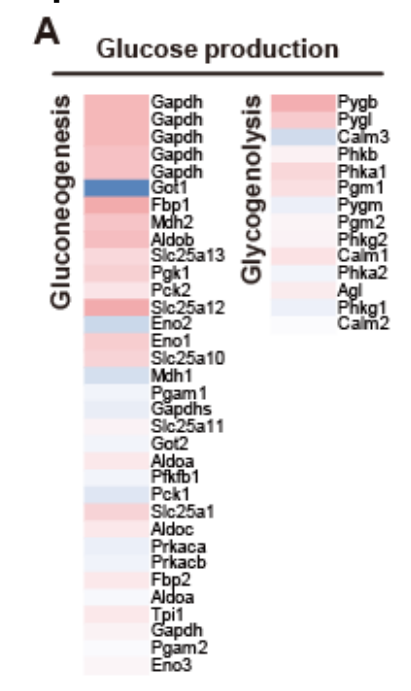

B
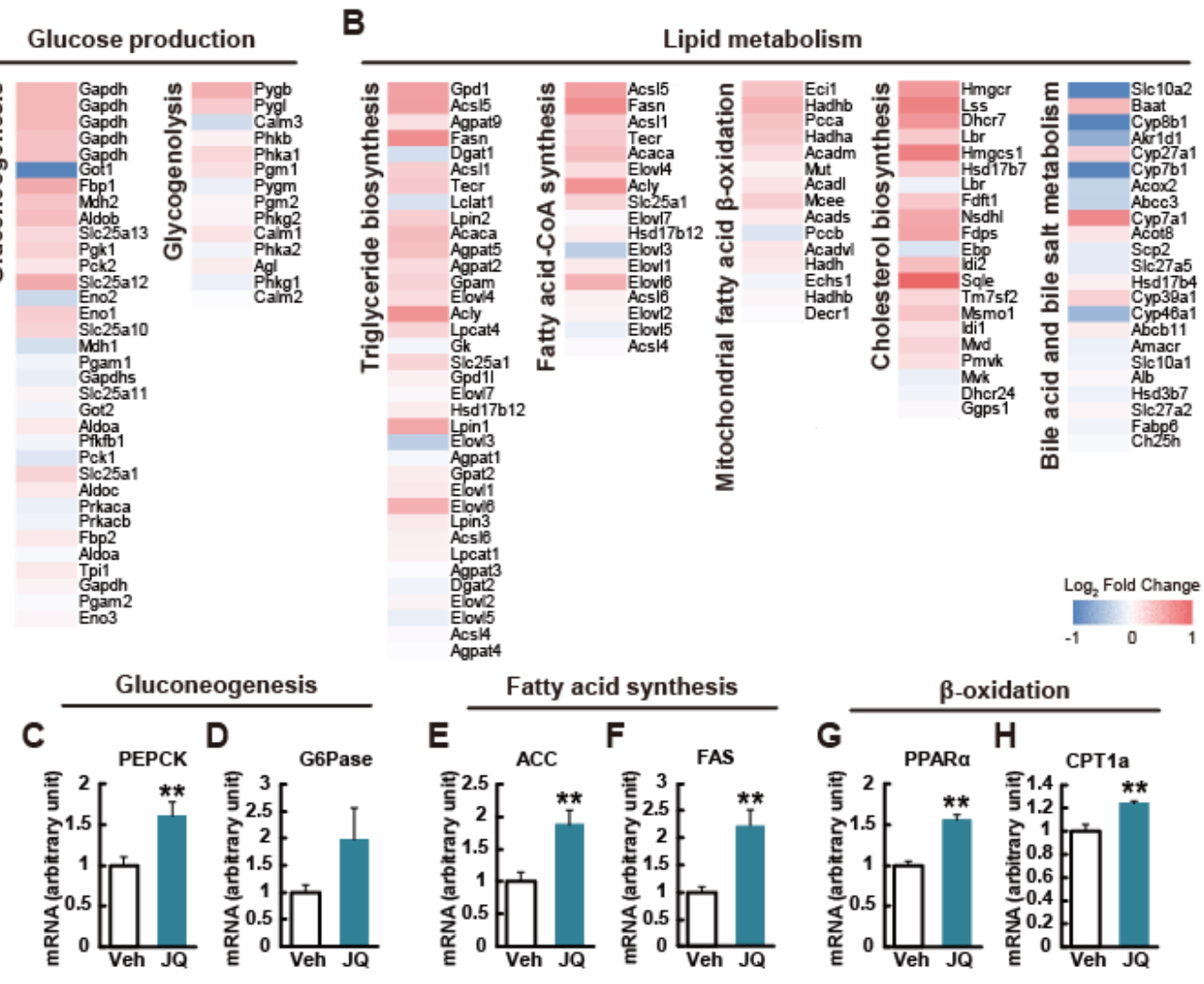

Cholesterol synthesis
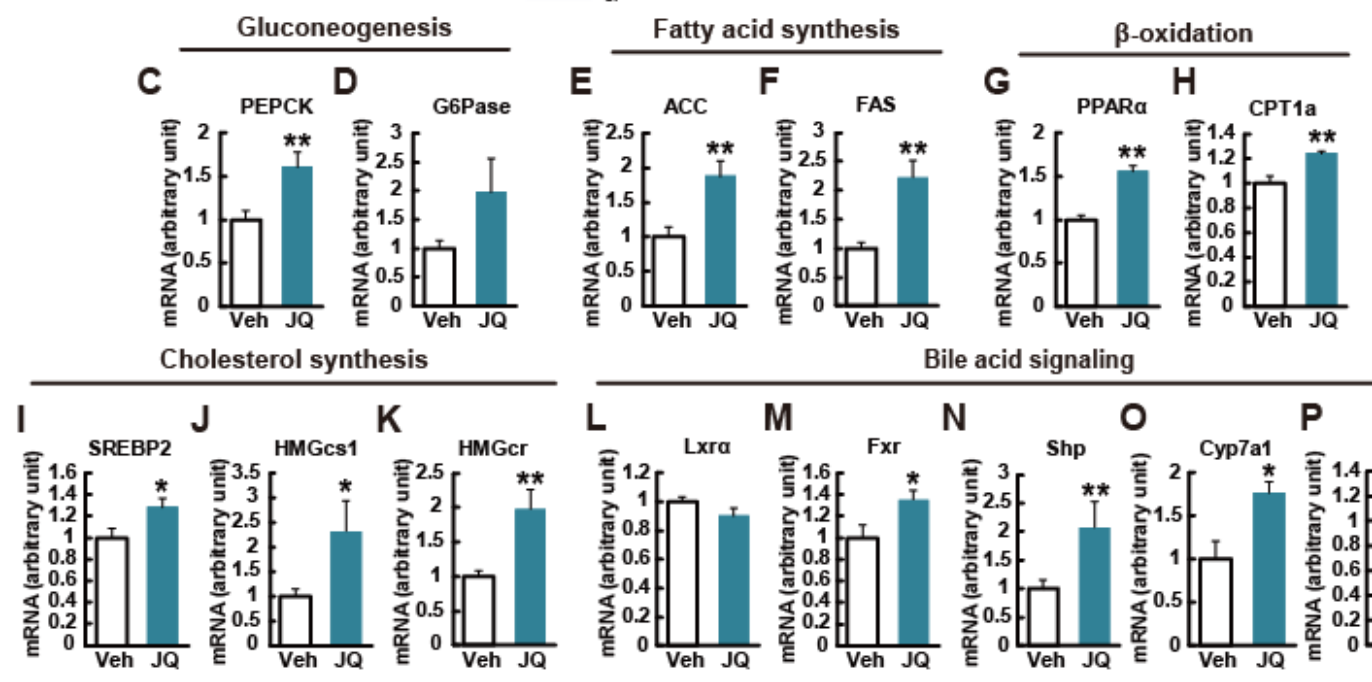

Bile acid signaling

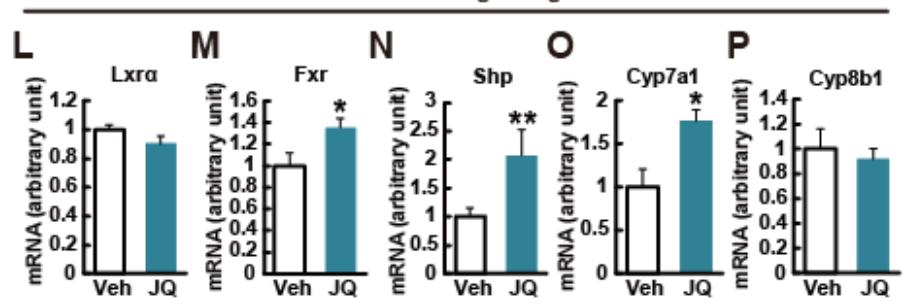

(A-B) Microarray analysis in the liver was performed in JQ-1-treated mice $(n=3)$. Heatmaps represent $\log _{2}$ fold changes in gene expression in pathways related to (A) glucose production, (B) lipid metabolism. Expression levels of genes related to (C, D) gluconeogenesis (Pepck (C) and G6Pase (D)), (E, F) fatty acid synthesis (Acc (E), and Fasn (F)), (G, H) $\beta$-oxidation (Ppara (G), and Cpt1a (H)), (I-K) cholesterol synthesis (Srebp2 (I), Hmgcs1 (J) and Hmgcr (K)) and (L-P) bile acid synthesis and signaling (Nr1h3 (LXRa) (L), Nr1h4 (FXR) (M), NrOb2 (SHP) (N), Cyp7a1 (O), and Cyp8b1 (P)) in the liver $(n=5-6)$. The levels were normalized by those of $R p / 13 .{ }^{*} P<0.05,{ }^{* *} P<0.01$, vs. vehicle-treated mice (Veh). ${ }^{*} P<0.05,{ }^{*} P<0.01$, vs. vehicle-treated mice (Veh). Data are expressed as means \pm SEM. 
Supplemental Figure 6. JQ-1 modulates liver and plasma lipids.

A

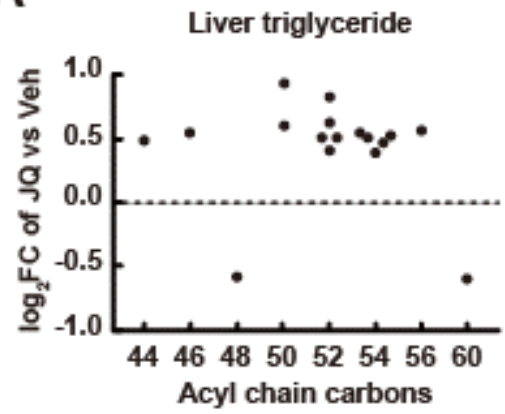

C
B

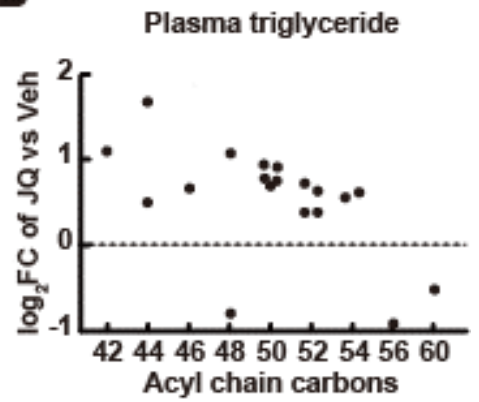

D

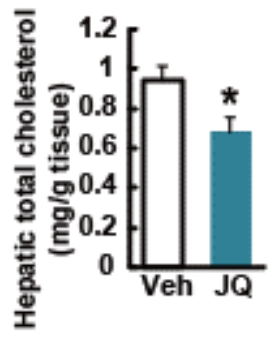

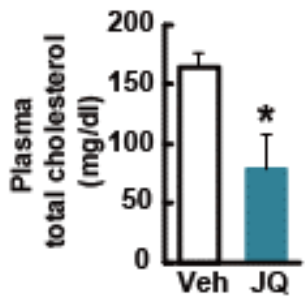

(A, B) Triglyceride species (FDR $<0.25)$ are upregulated in JQ-1 liver $(\mathbf{A})$ and plasma (B). X-axis indicates total acyl chain carbons, while Y-axis indicates log2 fold change (JQ/vehicle). (C, D) Hepatic (C) and plasma (D) total cholesterol levels in JQ-1 treated mice $(n=5-6) .{ }^{*} P<0.05, v s$. vehicle-treated mice (Veh). Data are expressed as means \pm SEM 
Supplemental Figure 7. JQ-1 decreases Reg4+ Paneth cells in the ileum.

A
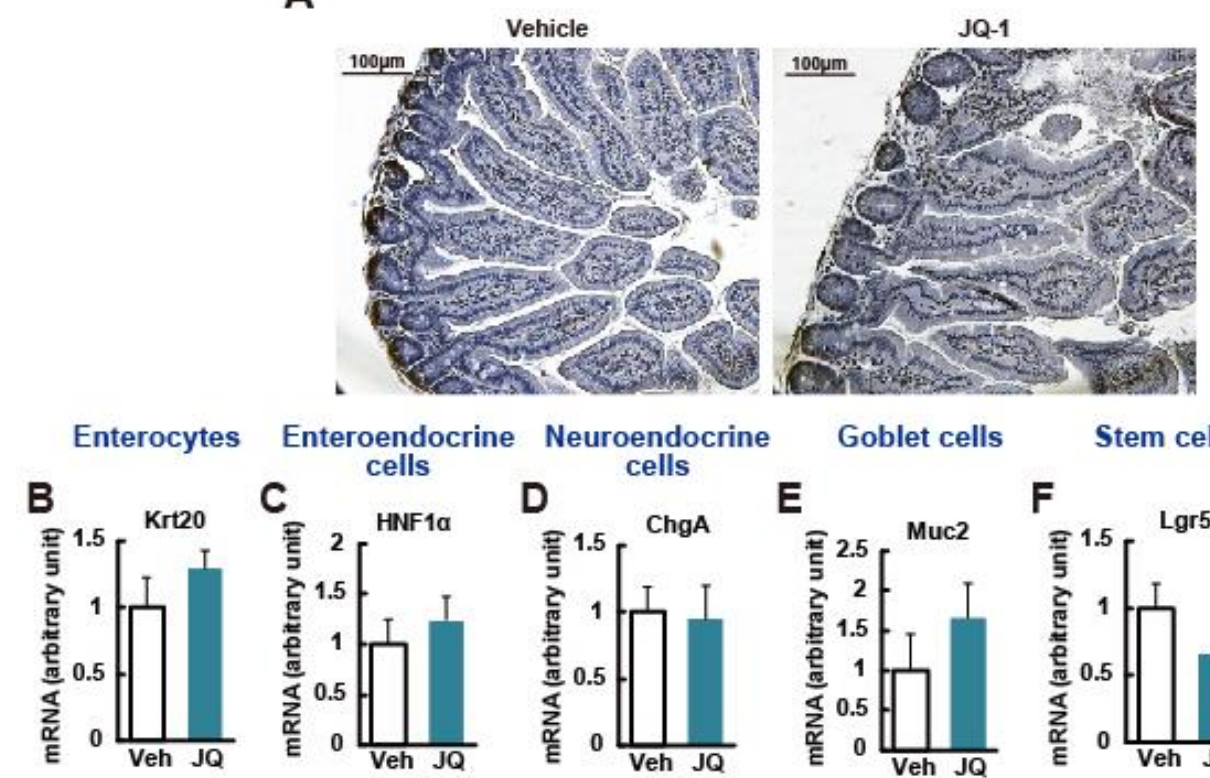

$\begin{gathered}\text { Enteroendocrine } \\ \text { cells }\end{gathered}$
$\begin{gathered}\text { Neuroendocrine } \\ \text { cells }\end{gathered}$

Goblet cells

Stem cells

Paneth cells
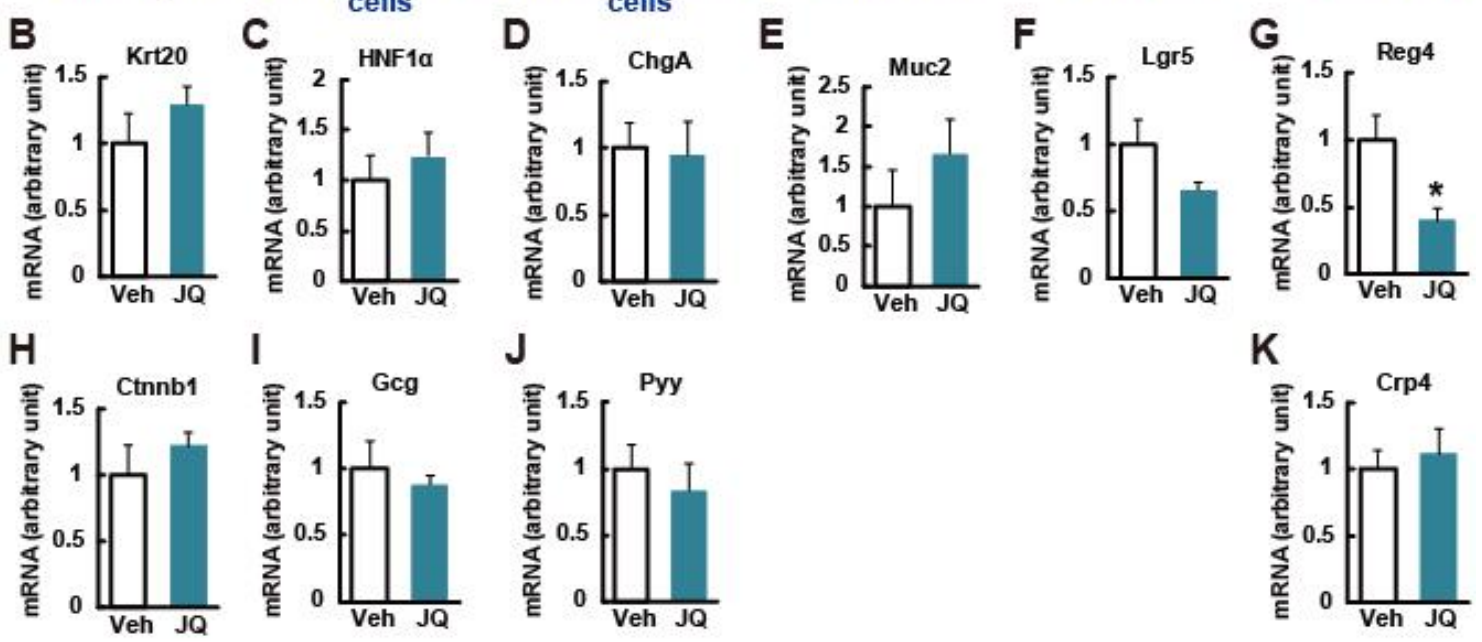

(A) Lysozyme staining of intestinal sections. Scale bar, $100 \mu \mathrm{m}$. (B-K) Gene expression levels of cell type marker genes. Krt20 (B) and Ctnnb1 (H) are Enterocyte markers, HNF1a (C) is an enteroendocrine cell marker, and the cells secrets Gcg (I). ChgA (D) is a neuroendocrine cell marker, and the cells secrets Pyy (J). Muc2 (E) and Lgr5 (F) are markers of goblet cell and stem cell, respectively. Reg4 (G) is a Paneth cell marker, and the cell secrets Crp4 (K). ${ }^{*} P<0.05$ vs. vehicle (Veh)-treated cells. Data are expressed as means \pm SEM. 
Supplemental Figure 8. Gluconeogenic gene expression levels were not changed by JQ-1 treatment in AAV-FGF19-treated mice.

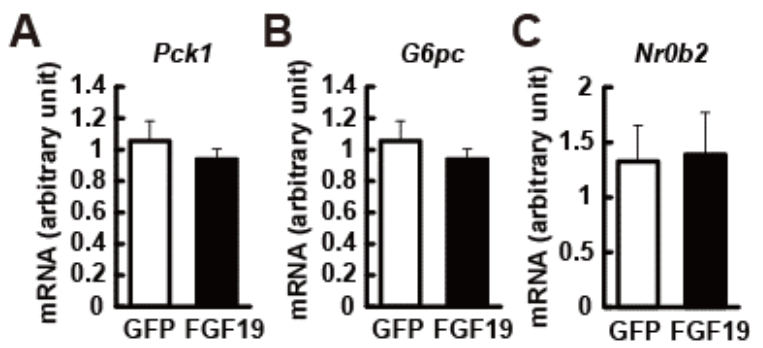

All animals were received JQ-1 treatment for 10 days. Gene expression levels of Pepck (Pck1) (A), G6pc (B), and Shp (NrOb2) (C) in the liver ( $n=5-6)$. Gene expression levels were normalized by those of $36 B 4$. Data are expressed as means \pm SEM. 
Supplemental Figure 9. Glucose intolerance caused by JQ-1 treatment was seen in intestine-specific Fgf15 knockout (Fgf15 $\left.{ }^{\mathrm{INT}-\mathrm{KO}}\right)$ mice.
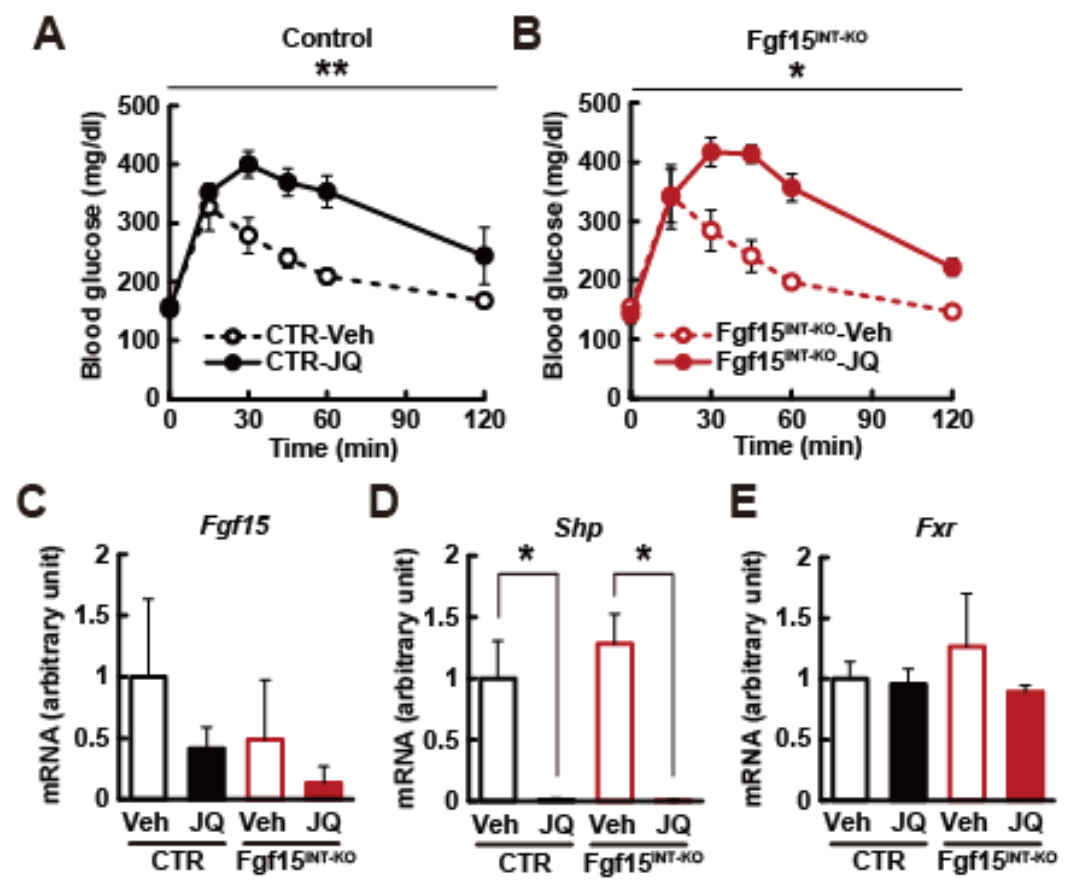

Control (CTR) and Fgf15 ${ }^{\text {INT-KO }}$ mice were treated with JQ-1 (50 mg/kg/day ip) for 8 days. (A, B) Blood glucose levels during ipGTT in control (CTR) (A) and Fgf15 $5^{\text {INT-KO }}$ (B) mice. (C-E) Gene expression levels of Fgf15 (C), Nr1h4 (Fxr) (D), NrOb2 (Shp) (E) in the liver $(n=3-8)$. Gene expression levels were normalized by those of 36B4. Data are expressed as means \pm SEM. ${ }^{*} P<0.05,{ }^{*} P<0.01$. 
Supplemental Figure 10. Expression of Fgf15 and Shp is reduced in ileum in mice with developmental exposure to maternal undernutrition.

A

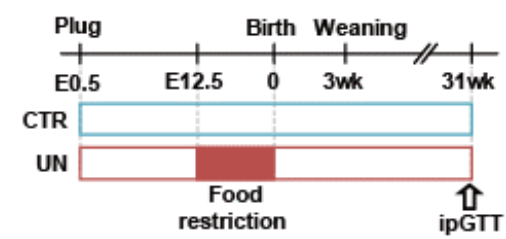

B

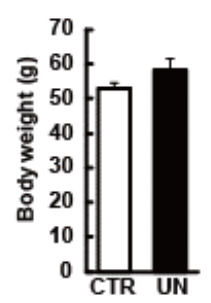

C
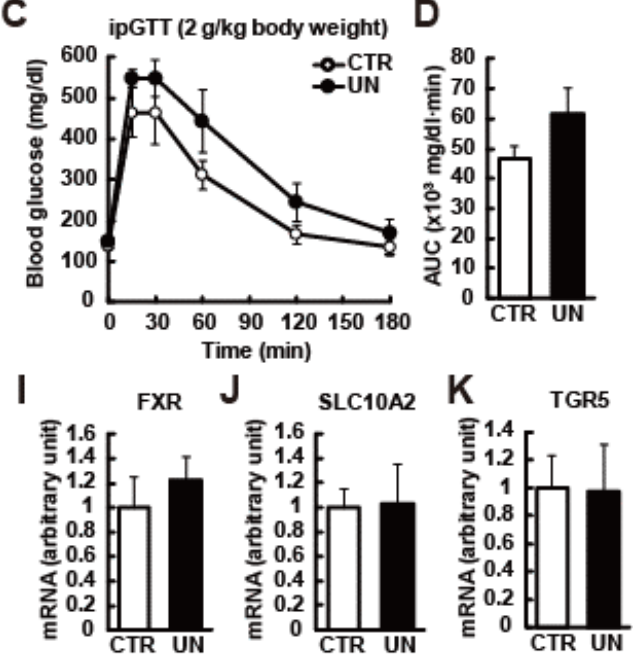

(A) Experimental design. Control animals (CTR) and their mothers were housed with ad libitum access to chow diet. Food intake of mothers of undernutrition animals (UN) was restricted from day 12.5 until delivery by $50 \%$ compared with that consumed by mothers of CTR. After delivery, mothers and pups of both groups received chow ad libitum. (B) Body weight of CTR and UN at 31-week-old $(n=4-5)$. (C, D) Blood glucose levels and area under the curve (AUC) during ipGTT $(n=4-5)$. (E-K) Expression levels of genes related to bile acid signaling in ileum (Fgf15 (E), NrOb2 (SHP) (F), Slc51a (OSTa) (G) and Slc51b (OST $\beta)(\mathbf{H})$ FXR (I), Slc10a2 (J) Tgr5 (K) ( $n=4-5)$. Expression levels were normalized by $R p / 13$ expression. ${ }^{*} P<0.05$, vs. CTR mice. Data are expressed as means \pm SEM. 